\title{
Willingness to Disclose Personal Information: How to Measure It?
}

\author{
Mindaugas Degutis ${ }^{1}$, Sigitas Urbonaviciuss ${ }^{1}$, Ignas Zimaitis ${ }^{1}$, Vatroslav Skare², Dalia Laurutyte1 \\ ${ }^{1}$ Vilnius University \\ Sauletekio av. 9, LT- 10222 Vilnius, Lithuania \\ E-mail.mindaugas.degutis@evaf.vu.lt; sigitas.urbonavicius@evaf.vu.lt; ignas.zimaitis@evaf.vu.lt; laurutyted@gmail.com \\ ${ }^{2}$ University of Zagreb, Faculty of Economics \& Business \\ Trg J.F. Kennedya 6, 10000 Zagreb, Croatia \\ E-mail.vskare@efzg.hr
}

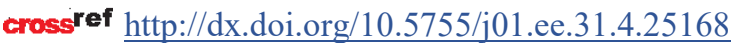

The study investigates a possibility of multidimensionality of a construct of willingness to disclose personal information (WTD). Willingness or unwillingness to disclose personal information has been a widely studied phenomenon as personal data is becoming increasingly important for many industries including marketing. Most of these studies treat the willingness to disclose personal information as a homogenous construct. In many cases it is measured by providing a number of personal information items and asking about the willingness to share them. Although recently there have been studies that find possible multidimensionality of the construct, most of them do not further elaborate this possibility. Therefore, we have adopted a scale used in many previous studies and made an attempt to test the hypotheses that would base the argument regarding the multidimensionality of this construct or even the possibility to consider several separate variables and constructs aimed at measuring the willingness to disclose personal data. This was achieved by using three antecedents of the willingness to disclose personal data - the perceived regulatory effectiveness, privacy awareness and disposition to value privacy - and comparing how they interact with different types of the willingness. This allowed to assess different relationship patterns between the antecedents and possible dimensions of the willingness to disclose personal information. We have employed Exploratory and Confirmatory Factor Analysis to check the homogeneity of the willingness to disclose personal information and Structural Equation Modelling to test the patterns of the relations. We have found that there is more than one separate dimension of WTD which means it could not be treated as a homogenous construct. Factorial analysis distinguishes three types of the willingness linked with three types of data: the willingness to disclose personal data that includes individual facts (profile data), social networking data and online browsing/purchasing data. The conclusion of multidimensionality is also supported by the differences in relationship patterns observed between the antecedents and the willingness to disclose personal information.

Keywords: Online Privacy; Sharing Personal Data; Privacy Awareness; Privacy Value; Perceived Regulatory Effectiveness.

\section{Introduction}

Predictive marketing based on consumer personal data analytics has become a common approach for many business companies and organizations around the world during the recent decades (Omer \& Levin, 2015). More and more companies, both internet and offline based, are trying to collect personal data of their consumers or visitors in order to use it for a variety of analytical and/or communication purposes (Paine Schofield \& Joinson, 2008). At the same time, consumers leave more and more personal information online (Boerman et al., 2018) hoping to increase the usability, convenience of the website or get other benefits. Many businesses use personal information for personalization of services and messages (Boerman et al., 2017; EstradaJimenez, 2017), be it advertising or political microtargeting.

Therefore, information privacy has become an increasingly important topic for academic research (Rohunen et al., 2018), as it plays an important role in the online purchasing process (Cosar et al., 2017). One of the major topics of this literature stream is about understanding what causes consumer willingness to disclose (WTD) personal information (Miltgen \& Smith, 2015). One type of antecedents is related with personal dispositions of consumers (e.g. their values (Anic et al., 2018), personality traits (Bansal et al., 2016), privacy attitudes and privacy experiences such as prior experience with privacy invasion (Malhotra et al., 2004; Xu et al., 2011), as well as cultural backgrounds (Gupta, Iyer, \& Weisskirch, 2010; Robinson, 2017). Another type of antecedents under analysis is related to socio-demographic characteristics (Weinberger et al., 2017), internet usage and habits (Akhter, 2014; Park, 2013). All these factors are based on personal characteristics of users. One more type of antecedents includes factors that could be named as situational factors, i.e. factors such as industry or company-specific variables, e.g. a general trust in the company or its reputation (Lwin et al., 2007; Xu et al., 2011). Situation-specific factors also include the perceived sensitivity, volume and relevance of information requested (Mothersbaugh et al., 2012), familiarity with the website and/or vendor, and incentives such as rewards offered for data disclosure. So, generally, privacy-related constructs (including but not limited to the antecedents of disclosure behaviour) can be dispositional, that is, belong to or be impacted by an individual's pre-existing attitudes, beliefs, tendencies, knowledge and skills, or situational - 
driven by context-dependent and "situation-specific privacy constructs" and their perceptions, e.g. related to a specific online company (Kehr et al., 2015).

Even though the antecedents of WTD personal information have received a prominent attention from scholars, the consequent construct of WTD personal information has not been yet extensively studied. There are several different scales used to measure the construct of the WTD personal information.

Some authors have measured the WTD personal information in general, leaving for respondents to decide which specific data types and items might be requested (Kehr et al., 2015; H. Li et al., 2011; Y. Li, 2014; Schoenbachler \& Gordon, 2002; Wakefield, 2013; T. Wang et al., 2016) while other researchers have referenced only data categories, such as financial information, personal health information and other (Bansal et al., 2016; Z. Wang \& Liu, 2014). Malhotra et al. (2004) have used a rather simple and convenient 4 item scale to measure a general disposition to disclose personal information. However, one of the most common approaches tends to list specific data types/items and ask the respondents to evaluate their disclosure intention on an item-by-item basis (Gupta et al., 2010; Heirman et al., 2013; Malheiros et al., 2013; Norberg et al., 2007; Robinson, 2017; Treiblmaier \& Chong, 2011; Walrave \& Heirman, 2012). This approach goes back to the measurements used by Phelps et al. (2000) and Sheehan and Hoy (2000). Some of these authors treat the scale as a single dimension measure of WTD personal information (Robinson, 2017; Gupta et al., 2010), while others find various dimensions and different behaviours of consumers related to them (Phelps, 2000, Heirman et al., 2013). This is justified by an increasing number of instances when personal data can be disclosed on the internet and a growing number of data types as well as multiple ways of data transfer. Therefore, the question of whether the willingness to disclose personal data is a homogenous construct is challenged. It seems quite possible that the WTD personal data varies depending on the types of data to be disclosed and, consequently, various instances of the willingness should be studied individually.

The aim of this study is to test the possible multidimensionality of the willingness to disclose personal data (WTD) construct. Additionally, we aim to test the hypotheses on different types of relations between the tested antecedents and various types/dimensions of the WTD construct.

\section{Theoretical Background}

One of the first examples measuring the WTD construct in the modern commerce context was a study by Phelps et al. (2000). The researchers used a 16-item scale and asked respondents to evaluate their willingness to disclose each 16 types of data on a 4-point scale (from 'always willing' to 'never willing'). Phelps et al. categorized 16 items into four groups: demographic characteristics, lifestyle, media usage habits and financial information. Nevertheless, this grouping was neither based on any type of statistical or other analytics, nor it was used for the subsequent analysis aimed to disclose their relations with antecedents or consequents. Therefore, although naming four groups of personal information, Phelps et al. (2000) treated the concept and the construct of WTD as a one-dimensional variable.
Gupta et al. (2010) examined consumer WTD in the US and India, adapted (shortened to 13 items) the scale used by Phelps et al. (2000) and deployed a 5-item scale to measure the willingness (from "not at all willing" to "very willing"). These researchers also treated the construct of the WTD as a homogenous unit.

The scales used by Gupta and Phelps were adapted by Robinson (2017) in his comparative study of Estonian and US consumers. He used a 7-item scale and expanded the list of items to 17 , including the ones related to the internet and ecommerce. In his analysis, he also used 6 sub-indices: Contact Information, Payment Information, Life History Information, Work-Related Information, Online Account Information, Financial/Medical History Info. He has concluded that there are some differences between Estonia and the US regarding the terms of the willingness to disclose different types of personal data (Robinson, 2017). These categories may be considered as sub-dimensions of the willingness, but the author did not elaborate on the possibility that there might be more than one separate type of willingness and separate constructs for the measurement of WTD.

This step was done by Heirman et al. (2013) who studied the willingness to disclose personal data to an internet site. These researchers proposed 4 separate subconstructs of WTD, namely: identity data, geographical information, contact data and profile data. They used a 7item scale to measure the willingness to provide each item of personal data and, after conducting factor analysis, confirmed the existence of 4 dimensions of WTD. They have also proved that there are differences in how an antecedent variable (namely, trust) influences various dimensions of the willingness to disclose personal data.

In this study, we have attempted to modify the existing WTD scale towards modern realities and situations when an individual may express a certain degree of the willingness to disclose personal data. Simultaneously, we avoid situations where an individual has no choice in disclosing certain types of data such as the necessity to provide a credit card or other banking information in order to perform a transaction. This leads to three types of personal data that are disclosed in a variety of instances: (a) personal data that discloses the basic demographic and contact information; (b) personal data that discloses social interactions of a person (account of social networks, communication engines) and (c) personal data that disclosed online behaviours and is collected automatically, based on a singletime permission (such as browsing history, location tracking). The first two types of data are provided by a person, but differ in terms of whether the data are linked with the parameters of an individual versus his/her social interactions; the third type differs by the form of its collection (automatic) and represents behavioural patterns. Consequently, these items may help to assess the three different types of WTD.

The WTD may be considered both as a dispositional (attitudinal) and situational variable. In this study, the dispositional aspect is considered, thus the three forms of willingness have to be related with the antecedents that are also dispositional by their nature. The three dispositional antecedents - the privacy awareness, disposition to value privacy and perceived regulatory effectiveness $(\mathrm{Xu}$ et al., 
2008) - have been widely studied in the context of privacy concerns and WTD and are included in the current study.

Individuals might demonstrate different inclinations towards certain privacy behaviours and various levels of disposition to value privacy which can be related to a disposition to value privacy as an inherent need and trait which reflects the extent to which a person is inclined to maintain their personal information in private as much as possible "across a broad spectrum of situations and persons" (Xu et al., 2008). The disposition to value privacy positively impacts online privacy concern and the perceived intrusive information gathering; a person who attributes higher value to his/her informational privacy is more likely to have a higher degree of serious concerns regarding personal data disclosure. The concerns of people with a high disposition to value privacy include issues not about the content of information, but also about how the personal data is collected, how it might be processed, i.e. some types of information gathering might be perceived as inappropriate and intrusive (Smith et al., 2011; Xu et al., 2008).

The disposition to value privacy is closely linked with one's awareness of privacy practices (privacy awareness). This dispositional factor reflects how an individual is aware of company practices, regulatory policies and privacy-related matters in the society (Xu et al., 2008). The awareness of privacy practices has been studied as an antecedent of disposition to value privacy and has been found to decrease the willingness to disclose personal information (Olivero and Lunt, 2004). Privacy has been generally considered as a natural right of individuals, both in theory and under national and international law (Smith et al., 2011). Therefore, the regulatory aspect of a person's privacy offline and online is important on societal and individual decision-making levels. The empirical findings support the importance of an individual's perceptions regarding privacy regulation as a higher perceived effectiveness was found to decrease privacy concern (Miltgen \& Smith, 2015) and to reduce the need for privacy protection behaviour (Lwin et al., 2007; Miltgen \& Smith, 2015). If consumers feel protected enough at a societal level, this reduces the need to put in individual efforts for privacy protection; people feel secure enough about the private data they provide (Miltgen \& Smith, 2015).

\section{Hypotheses}

Based on previous studies by Phelps et al (2010), Heirman et al. (2013), Robinson (2017) we assume that the willingness to disclose personal data is not a homogenous construct. We hypothesize that:

H1. The scale that measures the willingness to disclose personal data has more than one dimension.

We expect to find 3 dimensions of the willingness to disclose personal information: first - linked with the personal data that helps to identify a person and includes data items most frequently provided by an individual while browsing or purchasing online (name, address, e-mail, etc.); second related to the information about an individual's social networking (such as social account information) and the third - related with the information collected online automatically, once a permission is given (such as browsing history, location tracking, etc.). Correspondingly, this would mean three types of the willingness to disclose personal data: the willingness to disclose personal data (individual facts, WTD_PD_IND), the willingness to disclose personal data about social interactions (WTD_PD_SOC) and the willingness to disclose personal data that is collected online (WTD_OD). All the three types of willingness are supposed to have certain relations with the analysed antecedents: disposition to value privacy (DTVP), perceived regulatory effectiveness (PReg) and privacy awareness (PAware).

The disposition to value privacy is the closest dispositional variable to the WTD. Xu et al. (2008) defined the disposition to value privacy as an inherent need and trait which reflects the extent to which a person is inclined to maintain his personal information private "across a broad spectrum of situations and persons", thus it reflects the individual's need to preserve his personal space, the importance put on his or her privacy and personal information. $\mathrm{Xu}$ et al. (2008) identified the disposition to value privacy as a "cultural and personality characteristic" and argues that the information disclosure decision depends on this trait. It has the most direct influence on the willingness to disclose personal information of all types because of its nature. Additionally, it may moderate the influences of other factors. Therefore, the hypothesis follows:

$\mathrm{H} 2$. The disposition to value privacy will have a direct negative influence on all the three dimensions of the willingness to disclose personal data.

The perceived regulatory effectiveness is linked with the situations where somebody perceives disclosing his/her personal information and relates this the regulations of various forms of legislation, with an expectation that this information is protected (Miltgen \& Smith, 2015). The considered types of data most commonly include individual characteristics and behaviours. Therefore, the perceived regulatory effectiveness is supposed to directly influence the willingness to disclose contact and profile information and online data but will not necessarily be related to the disclosure of social networking information. The following hypothesis formulated:

H3. The perceived regulatory effectiveness will have a direct positive influence on the willingness to disclose personal data that include individual facts.

The awareness of privacy practices (privacy awareness) is a dispositional construct that reflects how an individual is aware of company practices, regulatory policies and privacy-related matters in the society (Xu et al., 2008). The individuals who are highly aware of the issues are more likely to "closely follow privacy issues, the possible consequences of a loss of privacy due to accidental, malicious, or intentional leakage of personal information, and the development of privacy policies" (Xu et al., 2008). The awareness of privacy practices has been found to be closely related with an individual's disposition to value privacy: it has been modelled as an antecedent of a disposition to value privacy and has been found to enhance this disposition in the e-commerce context. However, interestingly, it did not affect a disposition to value privacy in the social networking context (Xu et al., 2008). The privacy awareness is mainly linked with the disclosure of the information that reflects the individual demographic characteristics of a person. Therefore, it should only directly influence the willingness WTD_PD_IND: 
Mindaugas Degutis, Sigitas Urbonavicius, Ignas Zimaitis, Vatroslav Skare, Dalia Laurutyte. Willingness to Disclose Personal ...

H4. Privacy awareness will have a direct positive influence on the willingness to disclose personal data that include individual facts.

\section{Measurement Scales and Survey}

The survey data were collected in Lithuania by using CAWI survey and a self-administered questionnaire. The study included the scales that were developed and used in previous academic studies and that were demonstrating satisfactory reliability and validity. All the items were measured on a 1-7 Likert scale. A 3-item scale of disposition to value privacy was originally developed by $\mathrm{Xu}$ et al. (2008). They found Cronbach's to be $\alpha=0.88$. Later it was adapted by $\mathrm{Xu}$ et al. (2011), Li (2014). The perceived regulatory effectiveness scale ( 3 items, $\alpha=0.83$ ) was taken from Lwin et al. (2007) with a minor change that includes GDPR as an example. The privacy awareness scale (3 items) was taken from $\mathrm{Xu}$ et al. (2008). Later it was also used by $\mathrm{Xu}$ et al. (2011) and showed a good reliability $(\alpha=0.865)$. The willingness to disclose personal data was measured by a scale adopted from Gupta et al (2010) and Heirman et al. (2013) also used by Robinson (2017). It (with 14 items) showed a good reliability in earlier studies $(\alpha=$ 0.87 ) and was the most relevant recent scale of this type (Robinson, 2017). In this study, the original list of items was reduced from 17 to 9 by removing those that were linked with entirely technical issues that would not be understood by general population. However, the scale was amended with 5 items of personal data that are collected online automatically (on user consent).

The survey sample consisted of 439 respondents ranging from 18 to 69 years of age; the age group of 18-22 represented $32.1 \%$ of the respondents, those spanning $23-$ 35 covered $33.0 \%$; those 36 or older represented the remaining $34.9 \% .25 .1 \%$ of the respondents were male and $74.9 \%$ female. There were $54.9 \%$ of the respondents with bachelor degree or lower education qualifications and 45.1 $\%$ with master or higher.

One item was removed from the willingness to disclose the personal data scale because of the high skewness (2.532) and kurtosis (5.799). All other items were included into the exploratory factor analysis (maximum likelihood; Promax rotation with Kaiser normalization). Kaiser-Meyer-Olkin measure of sampling adequacy was 0.877 , Bartlett's test of sphericity was significant (0.000), approx. Chi-square 7401.378 and $\mathrm{df}=496$. The extracted factors explained 57.860 of the total variance. The dependent variable willingness to disclose the personal data appeared in three factors (see Table 1).

Table 1

Factor Loadings of Willingness to Disclose Personal Data (WTD)

\begin{tabular}{|l|c|c|c|}
\hline \multirow{2}{*}{} & \multicolumn{3}{|c|}{ Factor } \\
\cline { 2 - 4 } & WTD_OD & WTD_PD_IND & WTD_PD_SOC \\
\hline Full name & & 0.794 & \\
\hline Address & & 0.625 & \\
\hline Mobile phone & & 0.739 & \\
\hline E-mail & & 0.797 & \\
\hline Birthday date & & 0.459 & \\
\hline & \multicolumn{3}{|c|}{ Factor } \\
\hline LinkedIn account & \multicolumn{3}{|c}{0.759} \\
\hline
\end{tabular}

\begin{tabular}{|l|c|c|c|}
\hline \multirow{2}{*}{} & \multicolumn{3}{|c|}{ Factor } \\
\cline { 2 - 4 } & WTD_OD & WTD_PD_IND & WTD_PD_SOC \\
\hline $\begin{array}{l}\text { Facebook } \\
\text { account }\end{array}$ & & & 0.653 \\
\hline Skype account & & & 0.877 \\
\hline $\begin{array}{l}\text { Internet browsing } \\
\text { history and habits }\end{array}$ & 0.754 & & \\
\hline Geolocation data & 0.635 & & \\
\hline $\begin{array}{l}\text { Online } \\
\text { purchasing } \\
\text { history and habits }\end{array}$ & 0.926 & & \\
\hline $\begin{array}{l}\text { Information on } \\
\text { searched goods }\end{array}$ & 0.819 & & \\
\hline IP address & 0.543 & & $\mathbf{0 . 7 6 3}$ \\
\hline $\begin{array}{l}\text { Means of the } \\
\text { loadings: }\end{array}$ & $\mathbf{0 . 7 3 5}$ & $\mathbf{0 . 6 8 3}$ & \\
\hline
\end{tabular}

The first extracted factor - the willingness to provide online data (WTD_OD) - included the data that are linked with online activities but does not have to be provided by a person. It is required just to give a permission to track/record this type of the data, while the further processes are going automatically without a direct intervention of the internet user. The reliability of this scale was 0.854 . Two other factors represent personal data about the internet user. However, factor number 2 - the willingness to disclose personal data (individual facts, WTD_PD_IND) - includes the identification and demographic data of an individual, while factor number 3 the willingness to disclose personal data about social interactions (WTD_PD_SOC). The reliabilities of these scales were: $0 . \overline{8} 51$ and 0.853 , respectively. The reliability of scales that measured antecedents was also satisfactory: disposition to value privacy $\alpha=0.835$; perceived regulatory effectiveness $\alpha=0$. 746; privacy awareness $\alpha=0.829$; online privacy concern $\alpha$ $=0.901$;

A subsequent confirmatory factor analysis has been performed three times, with the same three same antecedents and each dependent variable separately. All the three models were robust and showed good fit (Table 2)

Table 2

Fit of the three Models (CFA)

\begin{tabular}{|l|l|l|l|}
\hline & WTD_PD_IND & WTD_PD_SOC & WTD_OD \\
\hline CMIN/DF & 1.242 & 1.242 & 1.350 \\
\hline TLI rho2 & 0.991 & 0.991 & 0.988 \\
\hline CFI & 0.993 & 0.993 & 0.991 \\
\hline RMSEA & 0.023 & 0.023 & 0.028 \\
\hline
\end{tabular}

On this basis, three causal models have been developed. In all the three instances, the presence of the common latent factor has been discovered, therefore the variables have been imputed considering its presence. All the three models demonstrated satisfactory fit (Table 3)

Table 3

Fit of the three Causal Models

\begin{tabular}{|l|l|l|l|}
\hline & WTD_PD_IND & WTD_PD_SOC & WTD_OD \\
\hline CMIN/DF & 3.472 & 1.041 & 2.862 \\
\hline TLI rho2 & 0.941 & 0.999 & 0.965 \\
\hline CFI & 0.990 & 1.000 & 0.994 \\
\hline RMSEA & 0.075 & 0.010 & 0.065 \\
\hline
\end{tabular}

This allowed to test the hypotheses. 


\section{Testing of the Hypotheses}

The first hypothesis H1 (The scale that measures the willingness to disclose personal data has more than one dimension) was tested on the basis of an exploratory factor analysis (Table 1) and a subsequent confirmatory factor analysis. The average factor loadings $(0.735,0.683,0.763)$ confirm the convergent validity, the correlations between factors (below 0.8) - discriminant validity (Table 4).

Table 4

\section{Correlation among Factors}

\begin{tabular}{|l|c|c|c|}
\hline & $\begin{array}{c}\text { WTD_PD_ } \\
\text { IND }\end{array}$ & $\begin{array}{c}\text { WTD_PD_ } \\
\text { SOC }\end{array}$ & WTD_OD \\
\hline WTD_PD_IND & 1.000 & 0.523 & 0.509 \\
\hline WTD_PD_SOC & 0.523 & 1.000 & 0.414 \\
\hline WTD_OD & 0.509 & 0.414 & 1.000 \\
\hline
\end{tabular}

Additionally, these three variables have a high reliability of their scales (Cronbach's $\alpha$ above 0.85). All this indicates that the three types of the willingness can be measured as three separate variables and allows to confirm $\mathrm{H} 1$.

Hypothesis $\mathrm{H} 2$ (the disposition to value privacy will have a direct negative influence on all the three dimensions of the willingness to disclose personal data) is tested on the basis of all the three causal models by checking the significance of the relation between the disposition to value privacy and corresponding types of WTD. In all the cases $\mathrm{p}=0.000$; WTD_PD_IND $\beta=-0.394$; WTD_PD_SOC $\beta=-$ 0.273 ; WTD_OD $\beta=-0.458$. Therefore, $\mathrm{H} 2$ is confirmed.

Hypothesis $\mathrm{H} 3$ (the perceived regulatory effectiveness will have a direct positive influence on the willingness to disclose personal data that includes individual facts) is tested on the basis of the causal model with the dependent variable WTD_PD_IND. In this case $\beta=0.097 ; p=0.045$. H3 is confirmed.

Hypothesis H4 (privacy awareness will have a direct positive influence on the willingness to disclose personal data that includes individual facts, the perceived regulatory effectiveness will have a direct positive influence on the willingness to disclose personal data that includes individual facts) is tested on the basis of the causal model with the dependent variable WTD_PD_IND. In this case $\beta=0.158$; $\mathrm{p}=0.004$. H4 is confirmed.

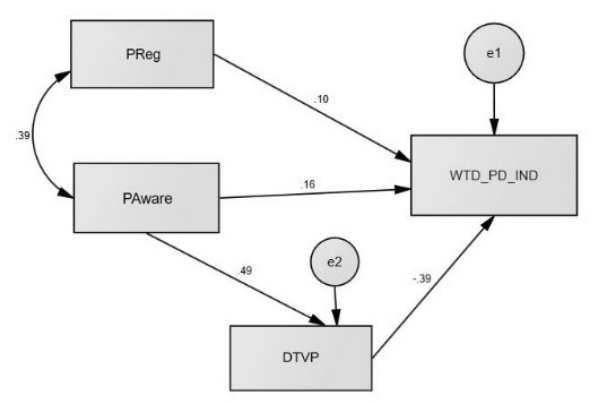

(a)

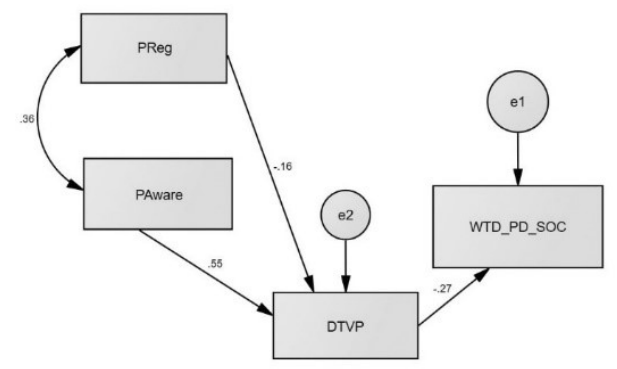

(b)

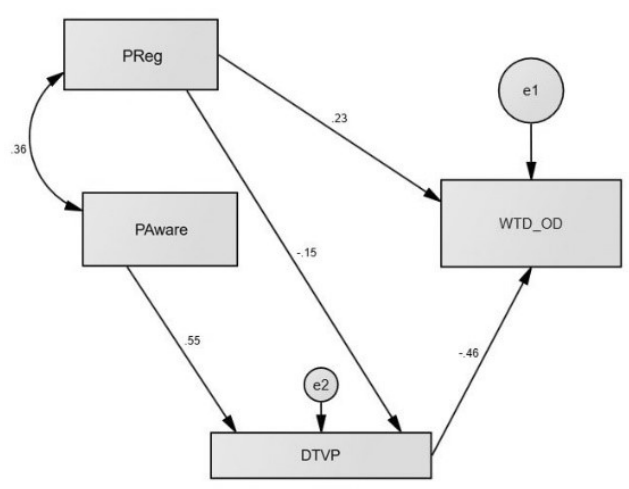

(c)

Figure 1. Causal Models

Although it was not included in the hypotheses, we aimed to disclose that the three antecedents influence all the three types of WTD, but this happens in different ways. These different relation patterns are presented in three different causal models (Figure 1).

The presence of different relation patterns supports the hypothesis about multidimensionality of the WTD construct, which may trigger discussion even about three separate variables that perhaps describe three types of data to be disclosed. Willingness that is linked with each type of data is directly influenced by DTVP, but PReg and PAware have direct impact just on one type of WTD - the one that considers disclosure of personal (individual) data, WTD_PD_IND. PReg and PAware have no direct impact on WTD_PD_SOC, and only PReg has direct impact on WTD_OD. Additionally, the different nature of the three types of the WTD is visible from the remarkable differences of the total effects of the three antecedents (table 5)

Table 5

Standardized Total Effects

\begin{tabular}{|l|c|c|c|}
\hline & PReg & PAware & DTVP \\
\hline WTD_PD_IND & 0.097 & -0.036 & -0.394 \\
\hline WTD_PD_SOC & 0.043 & -0.150 & -0.273 \\
\hline WTD_OD & 0.302 & -0.251 & -0.458 \\
\hline
\end{tabular}

\section{Discussion and Conclusions}

The findings of the current survey support a previous research carried out by Heirman et al. (2013). Factor analysis shows that there is more than one dimension in the 
willingness to disclose personal information construct. Heirman et al. (2013) found 4 separate dimensions while we found 3 dimensions instead of 4 . Probably, the difference is due to a larger number of items used in a survey conducted by Heirman et al. (2013). As mentioned previously, Heirman et al. (2013) distinguish 4 groups of personal data (although it is not based on any statistical model): identity data, geographical information, contact data and profile data. We find slightly different dimensions based on factorial analysis, namely personal contact and profile information, social networking data and internet usage and purchasing online information. This partially reflects the dimensions found by Heirman et al. (2013). Obviously, the consumers perceive personal data as a heterogenous phenomenon with all the consequences of this fact.

Not only the factor analysis shows multidimensionality of the WTD construct. T-test analysis shows that there is a significant difference between the average value of the three separate dimensions of willingness to disclose personal information. Test results (in both cases sig. $<0.001$ ) show that consumers are significantly more willing to disclose contact data and internet usage/purchasing information compared to social networking data. This supports the idea of difference in the perception of different types of personal information. It could be hypothesized that consumers perceive social networking data as more sensitive and intimate, therefore are consequently less willing to share it with others.

Further multidimensionality of WTD construct is supported by a different pattern of relationship between the antecedents and WTD. The disposition to value privacy has a negative relation with all the three dimensions of WTD, while the perceived regulatory effectiveness does not have any influence in case of social networking data (compared to a positive relationship in other two cases) and level of privacy awareness has positive relation with willingness to disclose personal data only in case of personal contact data disclosure (compared to no relationship in other two cases). Again, it could be hypothesized that consumers do not think that social networks could be effectively regulated by national or EU laws and, therefore, even better regulatory perception does not have a positive effect on the willingness to disclose this type of data. A positive relationship between privacy awareness (i.e. interest in privacy issues) and the willingness to disclose personal contact information shows that probably more educated consumers understand that this type of data is less sensitive compared to other types.

In the cases when the perceived regulatory effectiveness and privacy awareness have no direct impact on WTD, these variables influence WTD indirectly, via mediation of the disposition to value privacy. Additionally, these two factors may have both direct and indirect effects on WTD. However, the most important observation is not the strength of these influences, but the existence of three different causal models when three types of WTD are considered. This allows to additionally state that these three types of WTD may be assessed and analysed separately, since they represent different aspects of willingness to disclose personal data.

The multidimensionality of WTD issue is worth further investigation, probably including more items of personal information into factorial analysis. It might provide even more than 3 or 4 possible dimensions of the construct. More than that, additional justification might help concluding that it is possible to consider not just dimensions, but separate constructs and variables. Based on an additional theoretical evidence, these could help to better understand consumers' habits of dealing with personal data.

This project has received funding from the Research Council of Lithuania (LMTLT), Agreement No P-MIP-19-12.

\section{References}

Akhter, S. H. (2014). Privacy concern and online transactions: the impact of internet self-efficacy and internet involvement. Journal of Consumer Marketing, 31(2), 118-125. https://doi.org/10.1108/JCM-06-2013-0606

Anic, I. D., Budak, J., Rajh, E., Recher, V., Skare, V., \& Skrinjaric, B. (2018). Extended model of online privacy concern: what drives consumers' decisions? Online Information Review, 7(3), 41. https://doi.org/10.1108/OIR-10-2017-0281

Bansal, G., Zahedi, Fatemeh M., \& Gefen, D. (2016). Do context and personality matter? Trust and privacy concerns in disclosing private information online. Information \& Management, 53(1), 1-21. https://doi.org/10. 1016/j.im.2015.08.001

Boerman, S. C., Kruikemeier, S., \& Zuiderveen Borgesius F. J. (2017). Online behavioral advertising: A literature review and research agenda. Journal of Advertising, 46, 363-376. https://doi.org/10.1080/00913367.2017.1339368

Boerman, S. C., Kruikemeier, S., \& Zuiderveen Borgesius, F. J. (2018). Exploring Motivations for Online Privacy Protection Behavior: Insights From Panel Data. Communication Research. https://doi.org/10.1177/009 3650218800915

Coşar C., Panyi, K. \& Varga, A. (2017). Try Not to Be Late! - the Importance of Delivery Service in Online Shopping, Organizations and Markets in Emerging Economies, 8(2), 177-192. https://doi.org/10.15388/omee.2017.8.2.14186

Estrada-Jimenez, J., Parra-Arnau, J., Rodriguez-Hoyos, A., \& Forne, J. (2017). Online advertising: Analysis of privacy threats and protection approaches. Computer Communications, 100, 32-51. https://doi.org/10.1016/ j.comcom.2016.12.016

Gupta, B., Iyer, L. S., \& Weisskirch, R. S. (2010). Facilitating global e-commerce: A comparison of consumers willingness to disclose personal information online in the US and in India. Journal of electronic commerce research 11(1). 
Heirman, W., Walrave, M., Ponnet, K., \& Van Gool, E. (2013). Predicting adolescents' willingness to disclose personal information to a commercial website: Testing the applicability of a trust-based model. Cyberpsychology: Journal of Psychosocial Research on Cyberspace, 7(3). https://doi.org/10.5817/CP2013-3-3

Kehr, F., Kowatsch, T., Wentzel, D., \& Fleisch, E. (2015). Blissfully ignorant: the effects of general privacy concerns, general institutional trust, and affect in the privacy calculus. Information Systems Journal, 25(6), 607-635. https://doi.org/10.1111/isj.12062

Li, H., Sarathy, R., \& Xu, H. (2011). The role of affect and cognition on online consumers' decision to disclose personal information to unfamiliar online vendors. Decision Support Systems, 51(3), 434-445. https://doi.org/10.1016/ j.dss.2011.01.017

Li, Y. (2014). The impact of disposition to privacy, website reputation and website familiarity on information privacy concerns. Decision Support Systems, 57, 343-354. https://doi.org/10.1016/j.dss.2013.09.018

Lwin, May, Wirtz, J., \& Williams, J. D. (2007). Consumer online privacy concerns and responses: a power-responsibility equilibrium perspective. Journal of the Academy of Marketing Science, 35(4), 572-585. https://doi.org/10. 1007/s11747-006-0003-3

Malheiros, M., Preibusch, S., \& Sasse, M. A. (2013, June). "Fairly truthful": The impact of perceived effort, fairness, relevance, and sensitivity on personal data disclosure. In International Conference on Trust and Trustworthy Computing (pp. 250-266). Springer, Berlin, Heidelberg. https://doi.org/10.1007/978-3-642-38908-5_19

Malhotra, N. K., Kim, S., \& Agarwal, J. (2004). Internet Users' Information Privacy Concerns (IUIPC): The Construct, the Scale, and a Causal Model. Information Systems Research, 15(4), 336-355. https://doi.org/10.1287/isre.1040.0032

Miltgen, C. L., \& Smith, H. (2015). Exploring information privacy regulation, risks, trust, and behavior. Information \& Management, 52(6), 741-759. https://doi.org/10.1016/j.im.2015.06.006

Mothersbaugh, D. L., Foxx, W. K., Beatty, S. E., \& Wang, S. (2012). Disclosure Antecedents in an Online Service Context: The Role of Sensitivity of Information. Journal of Service Research, 15(1), 76-98. https://doi.org/10. $1177 / 1094670511424924$

Norberg, P. A., Horne, D. R., \& Horne, D. A. (2007). The privacy paradox: Personal information disclosure intentions versus behaviors. Journal of consumer affairs, 41(1), 100-126. https://doi.org/10.1111/j.1745-6606.2006.00070.x

Omer A., Levin D. (2015). Predictive marketing: easy ways every marketer can use customer analytics and big data. Wiley. Paine Schofield, C. B., \& Joinson, A. N. (2008). Privacy, trust, and disclosure online. In A. Barak (Ed.), Psychological aspects of cyberspace: Theory, research, applications (pp. 13-31). Cambridge, UK: Cambridge University Press.

Olivero, N., Lunt, P. (2004). Privacy versus willingness to disclose in e-commerce exchanges: the effect of risk awareness on the relative role of trust and control. Journal of Economic Psychology, 25(2), 243-262. https://doi.org/10. 1016/S0167-4870(02)00172-1

Park, Y. J. (2013). Digital Literacy and Privacy Behavior Online. Communication Research, 40(2), $215-236$. https://doi.org/10.1177/0093650211418338

Phelps, J., Nowak, G., \& Ferrell, E. (2000). Privacy Concerns and Consumer Willingness to Provide Personal Information. Journal of Public Policy \& Marketing, 19(1), 27-41. https://doi.org/10.1509/jppm.19.1.27.16941

Robinson, S. C. (2017). Disclosure of personal data in ecommerce: A cross-national comparison of Estonia and the United States. Telematics and Informatics, 34(2), 569-582. https://doi.org/10.1016/j.tele.2016.09.006

Robinson, S. C. (2018). Factors predicting attitude toward disclosing personal data online. Journal of Organizational Computing and Electronic Commerce, 28(3), 214-233. https://doi.org/10.1080/10919392.2018.1482601

Rohunen, A., Markkula, J., Heikkila, M., \& Oivo, M. (2018). Explaining Diversity and Conflicts in Privacy Behavior Models. Journal of Computer Information Systems, 1-16. https://doi.org/10.1080/08874417.2018.1496804

Sheehan, K. B., \& Hoy, M. G. (2000). Dimensions of privacy concern among online consumers. Journal of public policy \& marketing, 19(1), 62-73. https://doi.org/10.1509/jppm.19.1.62.16949

Schoenbachler, D. D., \& Gordon, G. L. (2002). Trust and customer willingness to provide information in database-driven relationship marketing. Journal of Interactive Marketing, 16(3), 2-16. https://doi.org/10.1002/dir.10033

Smith, H. J., Dinev, T., \& Xu, H. (2011). Information privacy research: an interdisciplinary review. MIS quarterly, 35(4), 989-1016. https://doi.org/10.2307/41409970

Treiblmaier, H., \& Chong, S. (2011). Trust and Perceived Risk of Personal Information as Antecedents of Online Information Disclosure. Journal of Global Information Management, 19(4), 76-94. https://doi.org/10. 4018/jgim.2011100104 
Mindaugas Degutis, Sigitas Urbonavicius, Ignas Zimaitis, Vatroslav Skare, Dalia Laurutyte. Willingness to Disclose Personal ...

Wakefield, R. (2013). The influence of user affect in online information disclosure. The Journal of Strategic Information Systems, 22(2), 157-174. https://doi.org/10.1016/j.jsis.2013.01.003

Walrave, M., \& Heirman, W. (2012). Adolescents, Online Marketing and Privacy: Predicting Adolescents' Willingness to Disclose Personal Information for Marketing Purposes. Children \& Society, 38. https://doi.org/10.1111/j.10990860.2011.00423.x

Wang, T., Duong, T. D., \& Chen, C. C. (2016). Intention to disclose personal information via mobile applications: A privacy calculus perspective. International Journal of Information Management, 36(4), 531-542. https://doi.org/10. 1016/j.ijinfomgt.2016.03.003

Wang, Z., \& Liu, Y. (2014). Identifying Key Factors Affecting Information Disclosure Intention in Online Shopping. International Journal of Smart Home, 8(4), 47-58. https://doi.org/10.14257/ijsh.2014.8.4.05

Weinberger, M., Zhitomirsky-Geffet, M., \& Bouhnik, D. (2017). Factors affecting users' online privacy literacy among students in Israel. Online Information Review, 41(5), 655-671. https://doi.org/10.1108/OIR-05-2016-0127

Xu, H., Dinev, T., Smith, H., \& Hart, Paul J. (2008). Examining the Formation of Individual's Privacy Concerns: Toward an Integrative View. ICIS 2008 Proceedings - Twenty Ninth International Conference on Information Systems. Available from internet: https://aisel.aisnet.org/icis2008/6

Xu, H., Dinev, T., Smith, J., \& Hart, Paul. (2011). Information Privacy Concerns: Linking Individual Perceptions with Institutional Privacy Assurances. Journal of the Association for Information Systems, 12(12), $798-824$. https://doi.org/10.17705/1jais.00281

The article has been reviewed.

Received in January 2020; accepted in October 2020.

This article is an Open Access article distributed under the terms and conditions of the Creative Commons Attribution 4.0 (CC BY 4.0) License (http://creativecommons.org/licenses/by/4.0/) 